\title{
Una Exploración de las Propiedades Espaciales Desde la Lógica de los Programas de Estímulo
}

\section{An Exploration of Spatial Properties Based on the Logic of Stimulus Schedules}

\author{
Felipe de Jesús Patrón Espinosa \\ Universidad Autónoma de Baja California
}

\author{
Víctor Hugo González Becerra \\ Universidad de Guadalajara
}

\author{
Mauricio Ortega González \\ Universidad Autónoma de Baja California
}

\author{
Carlos de Jesús Torres Ceja y Carlos Javier Flores Aguirre \\ Universidad de Guadalajara
}

\begin{abstract}
El objetivo de este trabajo fue explorar el efecto de variar la distancia espacial entre el bebedero y el conjunto luzpalanca sobre la ubicación espacial y la tasa de respuesta en ratas. Siguiendo la lógica de los programas temporales, se diseñó un experimento en el que una propiedad espacial (distancia) de los estímulos fue manipulada, mientras que las propiedades temporales se mantuvieron relativamente estables. El Grupo 1 fue expuesto a 3 fases en las que la distancia entre el bebedero y el conjunto luz-palanca fue aumentando. El Grupo 2 fue expuesto a condiciones similares, pero la distancia espacial fue disminuyendo. Al finalizar cada ciclo T de $30 \mathrm{~s}$ se entregó agua de forma contingente con una probabilidad de 1. Los sujetos fueron 8 ratas hembra de la cepa Wistar. Se emplearon cámaras de condicionamiento ampliadas en las que la ubicación espacial de los dispositivos de estimulación pudo cambiar. Los resultados mostraron que las ratas permanecieron más tiempo en la zona contigua al bebedero a pesar de que este cambiara de ubicación. También, se encontró que la tasa de respuesta mostró niveles bajos para todas las ratas a lo largo del experimento, no obstante, esta fue una función de la contigüidad espacial. Las pruebas no paramétricas de Wilcoxon y de U de Mann-Whitney fueron empleadas en el análisis estadístico. Se propone una serie de propiedades espaciales como la ubicación cardinal o la distribución geográfica que podrían ser estudiadas paramétricamente e integrarse en los programas de estímulo.
\end{abstract}

Palabras clave: programas temporales, dimensión espacial, ratas

\begin{abstract}
The aim of this paper was to explore the effect of varying the spatial distance between the liquid dispenser and the light-lever set on spatial location and response rate in rats. Based on the logic of temporally defined schedules, an experiment was designed in which a spatial property of the stimuli (distance) was manipulated while the temporal properties remained relatively stable. Group 1 was exposed to 3 phases in which the distance between the liquid dispenser and the light-lever set increased. Group 2 was exposed to similar conditions, but the spatial distance decreased as the phases progressed. T cycle length was $30 \mathrm{~s}$ and the probability of reinforcement was 1 . The reinforcer (water) was delivered at the end of the cycle. The subjects were 8 female Wistar rats. Extended conditioning chambers were used in which the stimulation devices could be placed in different locations. Results showed that rats stayed longer in the zone near the liquid dispenser even after it changed location. Also, the response rate showed low levels for all rats throughout the experiment; however, this was a function of spatial contiguity. Wilcoxon and Mann-Whitney U non-parametric tests were used in the statistical analysis. Some spatial properties such as cardinal location or geographic distribution are proposed, which could be studied parametrically and integrated into stimulus schedules.
\end{abstract}

Keywords: temporal schedules, spatial dimension, rats

Dentro del Análisis Experimental de la Conducta (AEC), los programas definidos temporalmente (Schoenfeld \& Cole, 1972; Schoenfeld, Cumming \& Hearst, 1956) pueden ser considerados como el ejemplar

Felipe de Jesús Patrón Espinosa y Mauricio Ortega González, Facultad de Ciencias Humanas, Universidad Autónoma de Baja California, Mexicali, México; Víctor Hugo González Becerra, Centro Universitario de los Valles, Universidad de Guadalajara, México; Carlos de Jesús Torres Ceja y Carlos Javier Flores Aguirre, Centro de Estudios e Investigaciones en Comportamiento, Universidad de Guadalajara, México.

La correspondencia relativa a este artículo debe ser dirigida a Felipe de Jesús Patrón Espinosa, Facultad de Ciencias Humanas, Universidad Autónoma de Baja California, Bulevar Castellón y Lombardo Toledano s/n, Conjunto Urbano Esperanza 21350, Mexicali, México. E-mail: felipe.patron@uabc.edu.mx 
paradigmático para el estudio de la relación entre la manipulación de las propiedades temporales de los estímulos y la actividad del organismo. Los programas temporales se han caracterizado por ser procedimentos en los que la variación paramétrica de las propiedades temporales en las que ocurren los estímulos es tomada como la variable que genera efectos sobre la respuesta del organismo.

Este tipo de procedimiento se planteó como una alternativa a los programas de reforzamiento propuestos por Ferster y Skinner (1957), afirmando que los programas temporales contaban con dos ventajas. La primera implica que con los programas temporales las categorías que clasifican los programas de reforzamiento se reducen de dos a una (tiempo), mostrando, así, ser más convenientes en términos de sencillez. Cabe recordar que los programas de reforzamiento se categorizan dependiendo del criterio que el organismo debe cumplir para producir el reforzador: número de respuestas para los programas de razón y un lapso transcurrido para los programas de intervalo. La segunda ventaja se relaciona con las reglas empleadas para la identificación de la respuesta a reforzar (intervalo-razón). De estas reglas, la que se eligió fue el tiempo, porque con esta se reduce la participación determinante del sujeto en el procedimiento experimental; en otras palabras, el sujeto no influye sobre la variable independiente como ocurre con los programas de razón, en los que la entrega del reforzador depende del número de respuestas (Schoenfeld \& Cole, 1972).

De forma tradicional, los programas temporales se componen por la repetición de un ciclo de tiempo (ciclo T) que, a su vez, se encuentra dividido por dos subciclos en los que es posible manipular la probabilidad de reforzamiento. El subciclo discriminativo $\left(\mathrm{t}^{\mathrm{D}}\right)$ es el segmento del ciclo $\mathrm{T}$ en el que la probabilidad de reforzamiento es mayor, mientras que el subciclo delta $\left(t^{\Delta}\right)$ es el segmento en el que la probabilidad de reforzamiento es menor. Así, la proporción que el subciclo t $\mathrm{t}^{\mathrm{D}}$ ocupa del ciclo $\mathrm{T}$ se denomina $\check{\mathrm{T}}$.

En conjunto con este procedimiento, Schoenfeld y Farmer (1970) han generado una propuesta teórica con respecto al desarrollo de los fenómenos conductuales. En esta se menciona que el investigador debe tomar en cuenta las propiedades continuas de la actividad del organismo en tiempo y espacio, pues la conducta ocurre tanto en coordenadas temporales como espaciales. A pesar de que en esta formulación es explícita la importancia de la dimensión espacial, diversos autores han señalado la existencia de un sesgo en el AEC, al existir mayor producción de estudios enfocados en la dimensión temporal, en comparación con estudios en los que se trata la dimensión espacial (Christie, 1996; Leising, Ruprecht \& Stahlman, 2014; McClearn \& Harlow, 1954; Rescorla \& Cunningham, 1979; Ribes, 1992). Este sesgo hacia el estudio de las propiedades temporales por encima de las propiedades espaciales ha sido identificado por autores externos al AEC, pero que también se interesan en el aprendizaje desde una perspectiva experimental; tal es el caso de las neurociencias (Nasser \& Delamater, 2016).

Lo anterior no quiere decir que en el AEC no existan estudios que se interesen por la dimensión espacial de los fenómenos conductuales. Es posible identificar estudios que comparten este propósito, pero su número es mínimo, comparado con los que cuentan con el objetivo de estudiar la dimensión temporal. Además, en la mayoría de estos estudios la dimensión espacial de los fenómenos conductuales se ha limitado a ser analizada como una variable dependiente. Por ejemplo, el procedimiento generalmente compartido por estos estudios implica que el investigador manipula alguna propiedad temporal de los estímulos (demora de reforzamiento, densidad de reforzamiento, duración del reforzador, entre otros) como una variable independiente, mientras que registra los cambios en las propiedades espaciales de la actividad del organismo como una variable dependiente (ubicación espacial, reorientación espacial, trayectorias, etc.). De este modo, la dimensión espacial es reducida a un efecto y no se concibe como un factor que determina el desarrollo de los fenómenos conductuales (para una revisión más completa de este tipo de procedimientos, consultar los siguientes estudios: Baum \& Rachlin, 1969; Pear, 1985; Pear \& Rector, 1979; Ribes \& Torres, 2000; Torres, Correa, \& Montes, 2006).

Dentro de los estudios interesados por la dimensión espacial en el AEC, ¿qué tipo de investigaciones se han realizado en las que se hayan explorado los efectos de manipular las propiedades espaciales de los estímulos sobre el condicionamiento operante? En relación con el aprendizaje discriminativo, se han estudiado los efectos de variar la contigüidad espacial entre el estímulo discriminativo y el manipulandum. Diferentes resultados sugieren que en este tipo de aprendizaje la ejecución de los sujetos es una función de incrementar la contigüidad espacial entre dichos estímulos (Iwai, Yaginuma \& Mishkin, 1986; Murphy \& Miller, 1955, 1959; Polidora \& Fletcher, 1964; Stollnitz, 1965; Yaginuma \& Iwai, 1986). En los últimos años, ha resurgido el interés por el estudio de la variable contigüidad espacial, pero, a diferencia de las investigaciones anteriores, estas se concentran en la distancia entre el lugar en el que se presenta la 
respuesta procuradora y la entrega del reforzador. Para investigar esto, Leising, Ruprecht, y Stahlman, (2014) expusieron a ratas a una condición en la que, dependiendo del ensayo, podían producir sacarosa al presionar una palanca que se encontraba a un lado del comedero o en otra palanca que se encontraba en la pared de enfrente al comedero. Los autores reportaron una función inversa entre la contigüidad espacial y la variabilidad de la respuesta de presión de palanca en términos de la desviación estándar de la tasa de respuesta; sin embargo, este efecto no fue del todo claro, pues el procedimiento empleado incluyó, en conjunto con la variable distancia espacial, la manipulación de la probabilidad de reforzamiento, limitando la posibilidad de identificar los efectos de dichas variables de forma aislada.

Desde aproximaciones de enfoque cognitivo, se ha empleado el laberinto acuático de Morris (1984) para estudiar los efectos de variar la distancia espacial entre señales (estímulos visuales o pistas) y la plataforma (meta) sobre el aprendizaje espacial. De forma general, los resultados de diferentes estudios han revelado que las señales desarrollan mayor saliencia si se encuentran ubicadas de forma cercana a la plataforma. Otro hallazgo consistente entre distintos estudios muestra que los organismos expuestos a este tipo de tarea permanecen más tiempo en el área o cuadrante en el que se encuentra la plataforma (Chamizo, Manteiga, Rodrigo \& Mackintosh, 2006; Chamizo \& Rodrigo, 2004; Maes, Fontanari \& Regolin, 2009; Rodrigo, Gimeno, Ayguasanosa \& Chamizo, 2014). Esta clase de efectos también se han encontrado en estudios en los que se emplean cámaras experimentales o de condicionamiento tradicionales y no laberintos de Morris (Blaisdell, Schroeder \& Fast, 2018; Wong, Leising \& Blaisdell, 2016).

En otra línea de investigación cognitiva, algunos autores se han interesado en responder cómo es que los animales logran anticipar el momento y el lugar en el que sus presas se encuentran regularmente disponibles; a este fenómeno se le ha denominado aprendizaje tiempo-lugar. Los resultados de estos estudios han mostrado que varias especies son capaces de anticipar el lugar y el momento correcto en el que el alimento estará disponible para ser producido después de un entrenamiento en el que el reforzador debió de ser producido en diferentes manipulandums siguiendo un orden secuencial (Biebach, Gordijn \& Krebs, 1989; Carr \& Wilkie, 1998; Gould, 1987; Harrison \& Breed, 1987; Mulder, Gerkema \& Van der Zee, 2013; Reebs, 1996; Saksida \& Wilkie, 1994).

La ubicación espacial en la que los estímulos ocurren es indispensable para el estudio del aprendizaje tiempo-lugar, y esta variable también se ha estudiado en el AEC, pero desde una aproximación diferente. Iversen, Sidman y Carrigan (1986) expusieron a monos Rhesus a una tarea de discriminación condicional con tres teclas que podían iluminarse de diferentes colores y que, al mismo tiempo, podían presentar líneas verticales u horizontales. En la Fase 1, el estímulo muestra se presentó siempre en la tecla de en medio y las teclas restantes fueron los estímulos comparativos. En la Fase 2, el lugar del estímulo muestra cambió a través de los ensayos. Los resultados revelaron que el porcentaje de respuestas correctas fue una función inversa de cambiar la ubicación espacial únicamente cuando los estímulos consistían de líneas verticales u horizontales. Desde hace algunos años, esta variable ha generado interés en el estudio de las relaciones de equivalencia (Sidman, 2000). Empleando procedimientos similares a los de Iversen et al. (1986), se ha encontrado que la ubicación espacial de los estímulos cumple una función importante para la emergencia de relaciones de equivalencia (Swisher \& Urcuioli, 2013, 2015).

La diferencia en la producción de investigaciones realizadas desde el AEC que se han enfocado en la dimensión temporal, en comparación con los estudios que se han enfocado en la dimensión espacial, fue la guía de este trabajo. Se propone la integración del estudio de las propiedades espaciales en el sistema propuesto por Schoenfeld et al. (1956) que tradicionalmente se ha caracterizado por el empleo de programas definidos temporalmente. Cabe recalcar que lo que se plantea en este estudio no es una metodología independiente o nueva; más bien, se parte de la lógica paramétrica propuesta por estos autores para abordar la dimensión espacial de la interacción organismo-ambiente, con la posibilidad de que en un futuro se puedan estudiar ambas dimensiones de forma simultánea y bajo la misma lógica.

En concreto, se presenta una perspectiva que pretende señalar la importancia de la dimensión espacial como determinante de los fenómenos conductuales (Ribes-Iñesta, Palacios, Hernández, \& León, 2018; Schoenfeld \& Farmer, 1970) y que, a la vez, fomente la interpretación de los hallazgos generados sobre la temática en el AEC siguiendo una lógica paramétrica. Para esto, se realizó un experimento con el objetivo de explorar los efectos de variar la distancia espacial entre el bebedero (agua) y el conjunto luz-palanca sobre la ubicación espacial y la tasa de respuesta en ratas. Debido a que este procedimiento es incipiente, pareció arriesgado proponer hipótesis específicas, pero se esperaban dos resultados generales: (a) la tasa de respuesta será una función de disminuir la distancia espacial entre el bebedero y el conjunto luz-palanca 
(de acuerdo a los hallazgos encontrados en los estudios de aprendizaje discriminativo) y (b) las ratas permanecerán más tiempo en las zonas cercanas al bebedero (de acuerdo a los resultados reportados al emplear el laberinto acuático de Morrris para estudiar el aprendizaje espacial).

\section{Método}

\section{Diseño}

Se empleó un diseño de replicación intrasujeto (Arnau, 1990) en la modalidad reversible multivariable (Castro, 1977) desde la perspectiva teórica y metodológica del AEC (Sidman, 1960/1973; Skinner, 1938, 1956). Los grupos se diferenciaron por el orden en el que los sujetos fueron expuestos a las condiciones experimentales a través de las fases, en otras palabras, un grupo fue expuesto a condiciones en las que la distancia espacial entre los dispositivos de estimulación fue disminuyendo, mientras que para el otro grupo esta fue aumentando.

\section{Sujetos}

Se emplearon ocho ratas hembra de la cepa Wistar de tres meses de edad y experimentalmente ingenuas al inicio del estudio. Las ratas se mantuvieron en el bioterio del Centro de Estudios e Investigaciones en Comportamiento de la Universidad de Guadalajara, México, donde se les brindaba atención veterinaria continua. Todas las ratas contaron con un peso corporal similar al inicio del experimento (198-275 g) y fueron sometidas a un régimen de privación de agua de 23 horas y 25 minutos al día. Después de cada sesión experimental tuvieron acceso libre y de forma individual a bebederos con agua durante cinco minutos en sus cajas habitación que se ubicaban en una colonia con temperatura (23-25 grados centígrados) y humedad controlada (45\%-55\%), así como ciclos día/noche de 12 horas. El alimento se encontró disponible mientras que las ratas se encontraron en sus cajas habitación.

\section{Aparatos}

Se empleó una cámara experimental de $85 \mathrm{~cm}$ de largo por $42 \mathrm{~cm}$ de ancho y $36 \mathrm{~cm}$ de alto con la opción para la instalación de distintos módulos de estímulo y respuesta en tres puertos ubicados en su pared operativa. El puerto central se encontraba a la mitad de la pared larga $(85 \mathrm{~cm})$ opuesta a la puerta por la que se introducían los sujetos. Los otros dos puertos se encontraban a los lados del puerto central a una distancia de $15 \mathrm{~cm}$. En cada puerto se pudo instalar, dependiendo de la fase experimental: (a) un estímulo visual que constó de un disco translúcido de $2,5 \mathrm{~cm}$ que podía iluminarse de color amarillo por medio de un foco de $28-\mathrm{V}$ ubicado a $9 \mathrm{~cm}$ del suelo, (b) un dispensador de agua de tipo gotero que entregó $0,3 \mathrm{~cm}^{3}$ de agua en cada activación (marca MED modelo ENV-201A) ubicado a $2 \mathrm{~cm}$ del suelo y (c) una palanca de respuesta (marca MED modelo ENV-110M) ubicada a $2 \mathrm{~cm}$ del suelo. Cabe señalar que, debido a que el dispensador de agua fue de tipo gotero, el agua producida no contó con un límite de tiempo para el acceso. Además, el bebedero contó con un sensor de respuestas de entrada de cabeza (marca MED modelo ENV-251L). Para el registro de la ubicación espacial de las ratas, se definieron tres zonas $(17 \mathrm{~cm}$ x $10 \mathrm{~cm})$ de acuerdo a su proximidad con los dispositivos de estímulo y respuesta (rectángulos 1, 2 y 3 en la Figura 1). La cámara se encontraba dentro de un cubículo de aislamiento acústico de color negro, mismo que contaba con dos ventiladores de $115 \mathrm{~W}$ que proporcionaron ventilación y ruido blanco. La luz general fue proporcionada por un foco de luz negra-ultravioleta de $20 \mathrm{~W}$. La programación y el registro de eventos se realizó mediante un equipo de cómputo que contó con una interfase y el software MED-PC IV para ambiente Windows. El análisis y representación gráfica de los datos se realizó a través de los software IBM SPSS Statistics 23®, TopScan 2.0®, G* power 3.1® y SigmaPlot 11.0®. Una cámara Panasonic WV-CP284® fue empleada para el registro en vídeo de las sesiones experimentales. En la Figura 1 se muestran las medidas de la cámara experimental y la disposición espacial en la que se instalaron los dispositivos para la fase con mayor contigüidad espacial. 


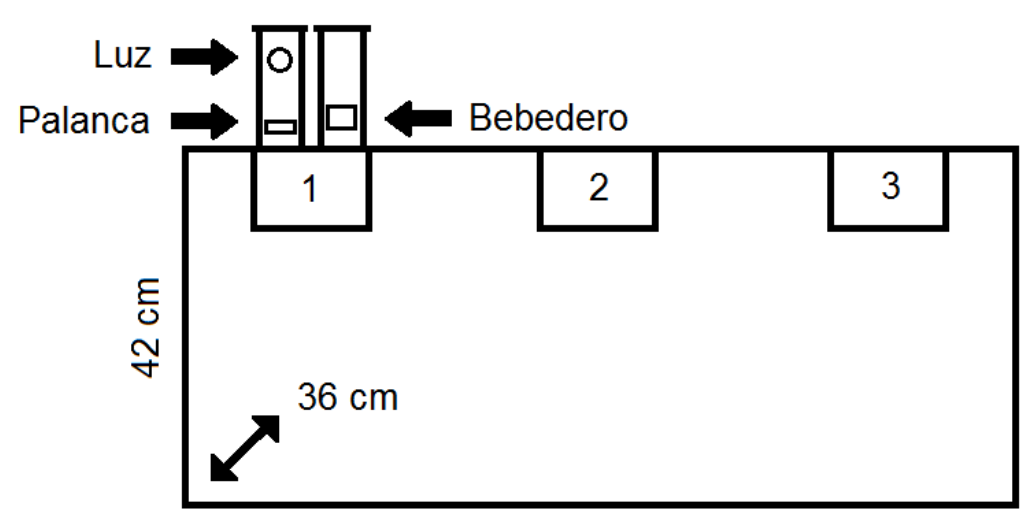

$85 \mathrm{~cm}$

Figura 1. Descripción esquemática de la cámara experimental. Los rectángulos con los números 1,2 y 3 representan las zonas próximas al puerto en el que pudieron instalarse los dispositivos de estímulo y respuesta. La imagen representa un caso en el que todos los dispositivos se encuentran en el puerto de la Zona 1 (Fase 1 para el Grupo 1 y Fase 3 para el Grupo 2).

\section{Procedimiento}

El estudio se realizó siguiendo las directrices de la Universidad de Guadalajara que cumplen con la Norma Oficial Mexicana NOM-062-ZOO-1999 para la producción, cuidado y uso de los animales de laboratorio, así como con la Norma Oficial Mexicana NOM-087-ECOL-SSA1-2002 para la clasificación y manejo de residuos biológicos. El empleo de los animales no humanos como sujetos para los experimentos fue aprobado por el Comité de Ética del Centro de Estudios e Investigaciones en Comportamiento de la Universidad de Guadalajara.

Los sujetos fueron entrenados a partir de la exposición a un programa de tiempo fijo de $30 \mathrm{~s}$ señalizado por el encendido de una luz. En conjunto con esto, se mantuvo activo un programa de reforzamiento continuo con el que se entregaba agua cada vez que el sujeto presionaba una palanca que se encontraba a un lado del bebedero y por debajo del estímulo visual (luz). Estas sesiones finalizaban después de 60 min o si se producían 100 reforzadores (agua). El entrenamiento finalizaba cuando se cumplía este criterio en tres sesiones consecutivas diarias. Durante este procedimiento, los dispositivos, como la palanca, el bebedero y la luz, fueron instalados en el puerto contiguo a la Zona 2. Después, se formaron dos grupos de cuatro ratas cada uno. Todos los sujetos fueron expuestos a un programa que entregaba agua cada $30 \mathrm{~s}(\mathrm{~T}=30 \mathrm{~s}) \mathrm{s}$ olo si la palanca de respuesta era presionada dentro del ciclo $(\breve{T}=1, P=1)$. Debido a que el interés del presente estudio se centró en los efectos de variar alguna propiedad espacial de los estímulos sobre la actividad de los organismos, se concluyó que entregar los reforzadores de forma inmediata a la respuesta procuradora implicaría que el momento en el que ocurre dicho estímulo dependía del sujeto y no de las manipulaciones programadas por el investigador. Considerando que para identificar los efectos de manipular una propiedad espacial es procedimentalmente práctico mantener los parámetros temporales estables, resulta contraproducente que el momento en el que se entrega el reforzador pueda variar de acuerdo a las respuestas del sujeto. Es por esto que para este experimento los reforzadores se entregaron de forma constante al final del ciclo T, procurando evitar que el momento en el que ocurra la entrega del agua dentro del ciclo dependa del sujeto. La disponibilidad de producción del reforzador $\left(\mathrm{t}^{\mathrm{D}}\right)$ fue señalada con la iluminación de un estímulo circular, mismo que se apagaba con la primera respuesta de presión de palanca del ciclo y se volvía a encender con el inicio del siguiente ciclo. Lo que distinguió entre grupos y fases fue el lugar (puerto) en el que los dispositivos se instalaron. Para el Grupo 1 la distancia espacial entre el bebedero y el conjunto luz-palanca fue aumentando a través de tres fases. Durante la Fase 1 todos los dispositivos (luz, palanca y bebedero) fueron instalados en el puerto de la Zona 1, es decir, la distancia entre los dispositivos fue nula. Para la Fase 2, únicamente el bebedero fue instalado en el puerto de la Zona 2, 
distanciándose $15 \mathrm{~cm}$ del conjunto luz-palanca. En la Fase 3 el bebedero fue instalado en el puerto de la Zona 3, distanciándose $30 \mathrm{~cm}$ del conjunto luz-palanca. Las fases para el Grupo 2 fueron similares pero en orden inverso, es decir, la distancia espacial fue disminuyendo con el cambio de las fases. Cada fase contó con 15 sesiones que finalizaban al transcurrir 60 ensayos que iniciaban cuando se colocaba a la rata en el área de en medio de la cámara. Al finalizar la Fase 3, todos los sujetos fueron expuestos a una fase de reversión de cinco sesiones en la que las condiciones experimentales fueron similares a las de la Fase 1 de su respectivo grupo. Se realizó una sesión al día en horario matutino de lunes a sábado. Para el registro de la ubicación espacial, cada rata fue filmada a lo largo de cada sesión experimental y dicho vídeo fue analizado por medio del software TopScan $2.0 \circledR$, cuantificando los segundos que la rata permaneció en las zonas representadas en la Figura 1. La tasa de respuesta fue calculada a partir de las respuestas de presión de palanca por minuto que las ratas presentaron durante la sesión.

\section{Análisis de Datos}

Los datos para cada uno de los sujetos fueron descritos de forma individual y se analizaron por medio de inspección visual sin empleo de estadística (Castro, 1977). Los datos agrupados fueron analizados con pruebas no paramétricas. Con el fin de comparar el tiempo que un mismo grupo permaneció en las zonas contiguas al bebedero y las zonas no contiguas (ubicación espacial), se empleó la prueba de rangos señalados y pares igualados de Wilcoxon y para las comparaciones entre grupos se empleó la prueba $U$ de Mann-Whitney. En adición, para comparar los cambios intragrupales en las tasas de respuesta dependiendo de la distancia espacial entre el bebedero y el conjunto luz-palanca (Fase 1, 2 y 3), se empleó la prueba de Friedman. Como análisis post hoc se empleó la prueba de Dunn-Bonferroni. A este análisis se agregó la prueba Wilcoxon para comparar las condiciones con valores extremos en lo que respecta a la distancia espacial entre los dispositivos de estimulación (Fase 1 y Fase 3). Para comparar los cambios en la tasa de respuesta entre grupos se empleó la prueba $U$ de Mann-Whitney. Para todas las pruebas el nivel de significación fue 0,05 (Pagano, 2011). El tamaño del efecto para cada prueba se calculó por medio del software $G^{*}$ power $3.1 \circledR$, excepto para la prueba de Friedman, que se calculó con el coeficiente de concordancia de Kendall $(W)$.

\section{Resultados}

En la Figura 2 se muestra la ubicación espacial en términos del tiempo promedio (segundos) que las ratas del Grupo 1 permanecieron en las Zonas 1, 2 y 3 a través de bloques de cinco sesiones. Los resultados revelaron que todas las ratas de este grupo permanecieron más tiempo en la zona espacialmente contigua al puerto en el que se instaló el bebedero a través de las fases. Como pudo observarse, la tendencia a permanecer en la zona cercana al bebedero varió conforme este fue instalado en los diferentes puertos. En otras palabras, en la Fase 1 las ratas permanecieron más tiempo en la Zona 1, en la Fase 2 permanecieron más tiempo en la Zona 2, en la Fase 3, en la Zona 3 y en la fase de reversión, en la Zona 1. Para la zona contigua al bebedero las ratas mostraron permanencias próximas a $1000 \mathrm{~s}$ o mayores, mientras que para el resto de las zonas las permanencias no superaron los $200 \mathrm{~s}$. 


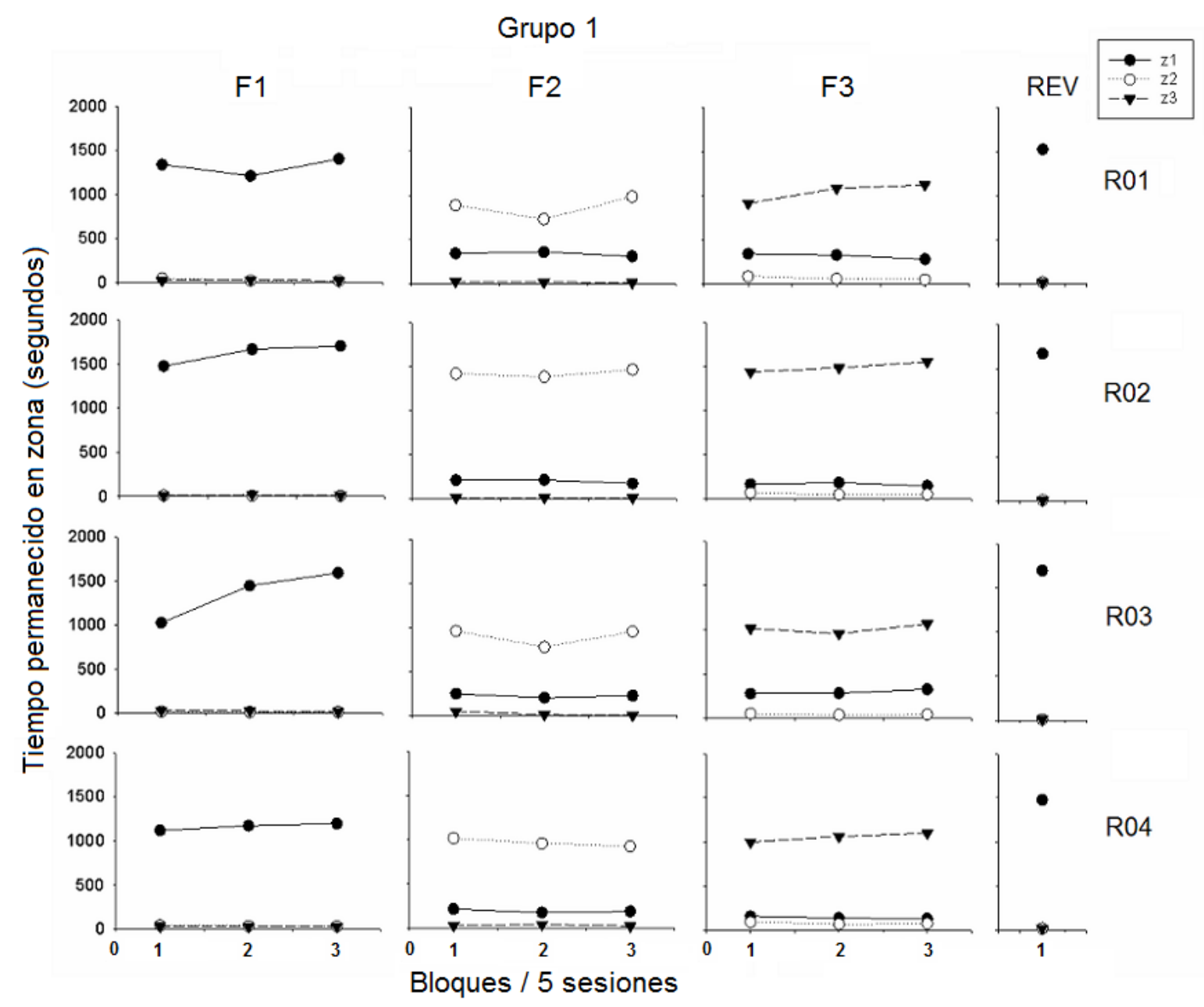

Figura 2. Tiempo promedio permanecido por las ratas del Grupo 1 en las Zonas 1, 2 y 3 a través de bloques de cinco sesiones. Para la fase de reversión solo se tomaron en consideración las primeras cinco sesiones. $\mathrm{R}$ es rata, $\mathrm{Z}$ es zona, $\mathrm{F}$ es fase y REV es reversión.

En la Figura 3 se muestra la ubicación espacial en términos del tiempo promedio (segundos) que las ratas del Grupo 2 permanecieron en las Zonas 1, 2 y 3 a través de bloques de cinco sesiones. Los resultados mostraron un efecto similar a lo observado para el Grupo 1, pero en orden inverso, es decir, en la Fase 1 las ratas permanecieron más tiempo en la Zona 3, en la Fase 2 permanecieron más tiempo en la Zona 2, en la Fase 3, en la Zona 1 y en la fase de reversión, en la Zona 3. El tiempo que las ratas permanecieron en la zona contigua al bebedero alcanzó valores cercanos a los $1000 \mathrm{~s}$ o mayores, pero el tiempo permanecido en las otras dos zonas fue menor que $200 \mathrm{~s}$. 


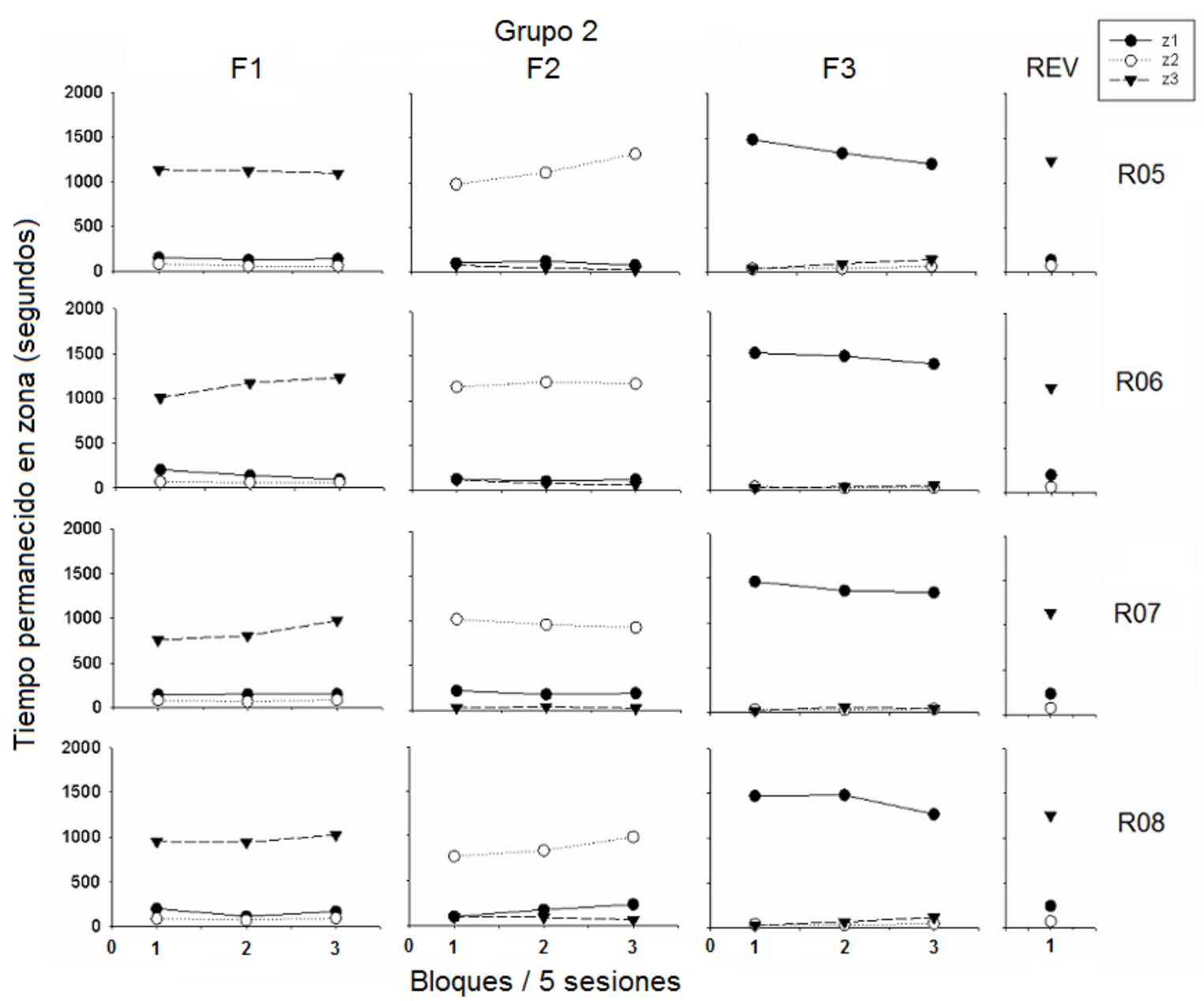

Figura 3. Tiempo promedio permanecido por las ratas del Grupo 2 en las Zonas 1, 2 y 3 a través de bloques de cinco sesiones. Para la fase de reversión solo se tomaron en consideración las primeras cinco sesiones. $\mathrm{R}$ es rata, $\mathrm{Z}$ es zona, $\mathrm{F}$ es fase y REV es reversión.

En la Figura 4 se muestra la media de la ubicación espacial de acuerdo al tiempo permanecido en la zona contigua al bebedero (ZB+) y en las dos zonas apartadas del bebedero (ZB-) para el Grupo 1 (panel superior) y el Grupo 2 (panel inferior) a través del experimento. Debido a que el bebedero fue cambiando de ubicación dependiendo de las fases, para la categoría ZB+ se tomó en consideración el tiempo que las ratas permanecieron en la zona contigua al bebedero en el que se entregó el agua, independientemente del puerto en el que se encontrara instalado. Los datos sobre la permanencia obtenidos de las otras dos zonas en las que no se encontraba el bebedero fueron incluidos en la categoría ZB-. 


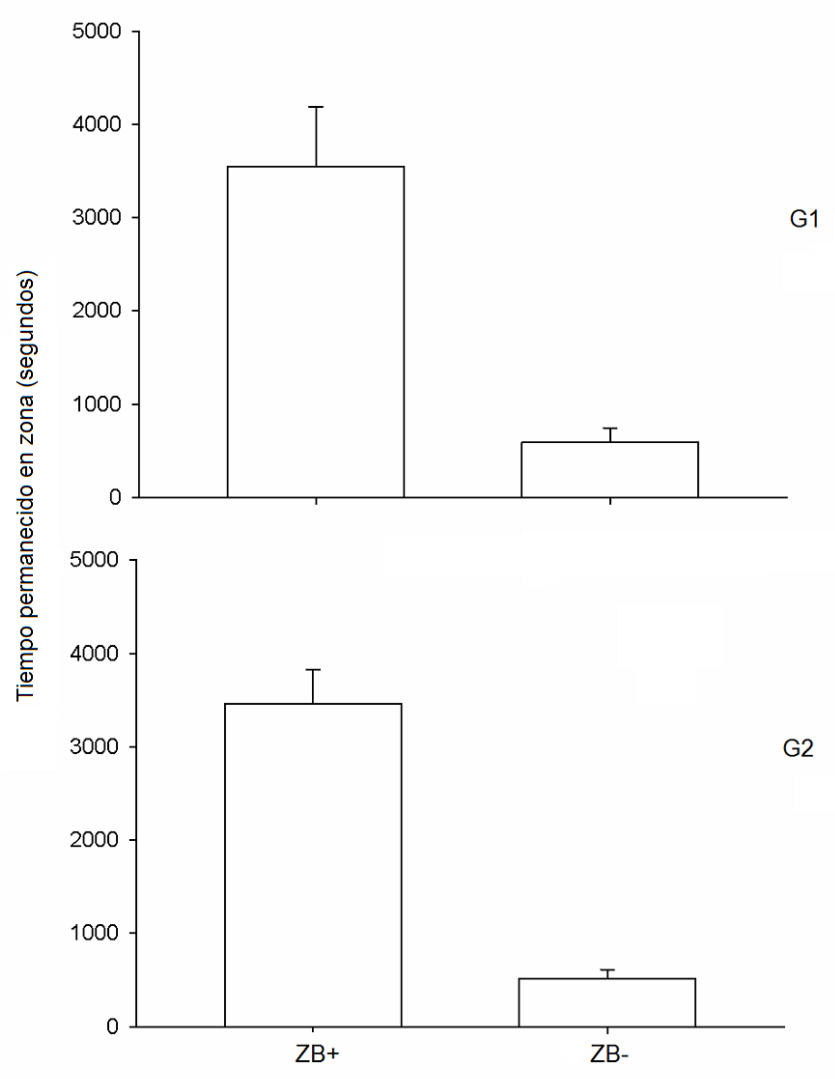

Figura 4. Tiempo promedio grupal permanecido en la zona contigua al bebedero (ZB+) y en las dos zonas discontiguas al bebedero (ZB-) para el Grupo 1 (panel superior) y el Grupo 2 (panel inferior). Las barras de error representan la desviación estándar.

Se empleó la prueba de Wilcoxon con el propósito de identificar diferencias en el tiempo permanecido en la zona contigua al bebedero y las zonas discontiguas de forma independiente para el Grupo 1 (panel superior) y el Grupo 2 (panel inferior). En el Grupo 1 se encontraron diferencias en el tiempo permanecido en ZB+ $(M d n=3348,520)$ y ZB- $(M d n=568,365), Z(N=4)=-6,736, p=0,001, d=5,100$. Por su parte, para el Grupo 2 también se encontraron diferencias en el tiempo permanecido en ZB+ $(M d n=3423,825)$ y ZB$(M d n=511,435), Z(N=4)=-6,736, p=0,001, d=8,914$. Al emplear la prueba $U$ de Mann-Whitney para comparar el tiempo permanecido por ambos grupos en ZB+ no se encontraron diferencias, $Z(N=8)=-0,441$, $p=0,659$; sin embargo, sí se encontraron diferencias al comparar el tiempo permanecido en ZB- para el Grupo $1(M d n=568,365)$ y el Grupo $2(M d n=511,435), Z(N=8)=-2,404, p=0,016, d=0,566$.

En la Figura 5 se muestra la tasa de respuesta (presión de palanca) media para el Grupo 1 a través de bloques de cinco sesiones. Para la fase de reversión solamente se tomaron en consideración las primeras cinco sesiones. Durante la Fase 1, la tasa de respuesta para las Ratas 02 y 03 fue una función del transcurso de las sesiones, alcanzando valores cercanos a 7 respuestas al final de la fase. La Rata 01 mostró tasas de respuesta que se mantuvieron relativamente estables en un rango entre 2 y 6 respuestas. Por su parte, la Rata 04 mostró tasas que no superaron las 4 respuestas a lo largo de la fase. En la Fase 2, las tasas de respuesta para las Ratas 01, 02 y 03 se mantuvieron relativamente estables en un rango entre 2 y 6 respuestas. La Rata 04 presentó un incremento en el Bloque 1, alcanzando una tasa cercana a 4 respuestas; sin embargo, estos valores disminuyeron conforme la fase transcurrió. En la Fase 3 se observaron tasas de respuesta mayores en comparación con la Fase 2; empero, esta diferencia fue tenue, pues todas las ratas presentaron tasas entre un rango de 4 y 8 respuestas. Durante esta fase, las Ratas 01, 02 y 03 alcanzaron tasas de respuesta mayores en comparación con la Rata 04. En la fase de reversión se 
observó una ligera tendencia al decremento en la tasa de la Rata 03, con valores cercanos a 5 respuestas. Por su parte, las tasas para el resto de las ratas se mantuvieron estables, es decir, con valores similares a los mostrados en el último bloque de la Fase 3.

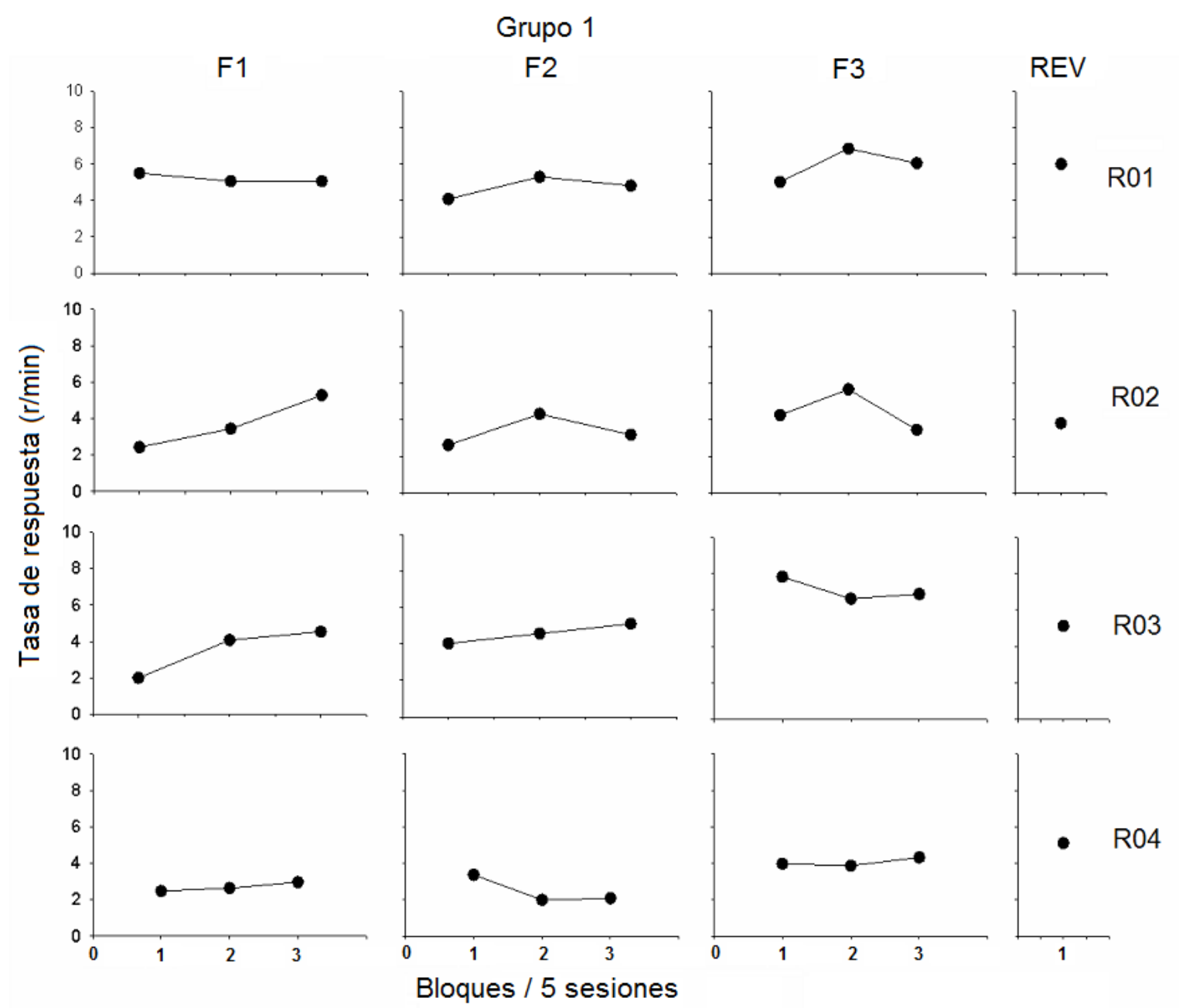

Figura 5. Tasa de respuesta promedio (respuestas por minuto) para las ratas del Grupo 1 a través de bloques de cinco sesiones. Para la fase de reversión solo se tomaron en consideración las primeras cinco sesiones. $\mathrm{R}$ es rata, $\mathrm{F}$ es fase y REV es reversión.

En la Figura 6 se muestra la tasa de respuesta (presión de palanca) media para el Grupo 2 a través de bloques de cinco sesiones. Para la fase de reversión solamente se tomaron en consideración las primeras cinco sesiones. En la Fase 1, todas las ratas presentaron tasas próximas a cero y con el transcurso de las sesiones estos valores no superaron las 2 respuestas. En la Fase 2, se observó un incremento progresivo en las tasas de todas las ratas conforme transcurrieron las sesiones, alcanzando valores cercanos a 4 respuestas en el Bloque 3. Durante la Fase 3, las ratas presentaron tasas de respuestas mayores en comparación con las de la Fase 2. Las Ratas 07 y 08 alcanzaron tasas cercanas a 6 respuestas; no obstante, también se observó una tendencia al decremento al final de esta fase. Las Ratas 05 y 06 mostraron tasas ligeramente más altas en comparación con la Fase 2, pero sus valores no superaron las 4 respuestas en la mayoría de los bloques. Finalmente, en la fase de reversión, las Ratas 05, 06 y 08 mostraron tasas que se mantuvieron relativamente estables, en comparación con las tasas observadas en el Bloque 3 de la Fase 2. Por su parte, la Rata 07 mostró la tasa más alta de su grupo en todas las fases: 8 respuestas. 


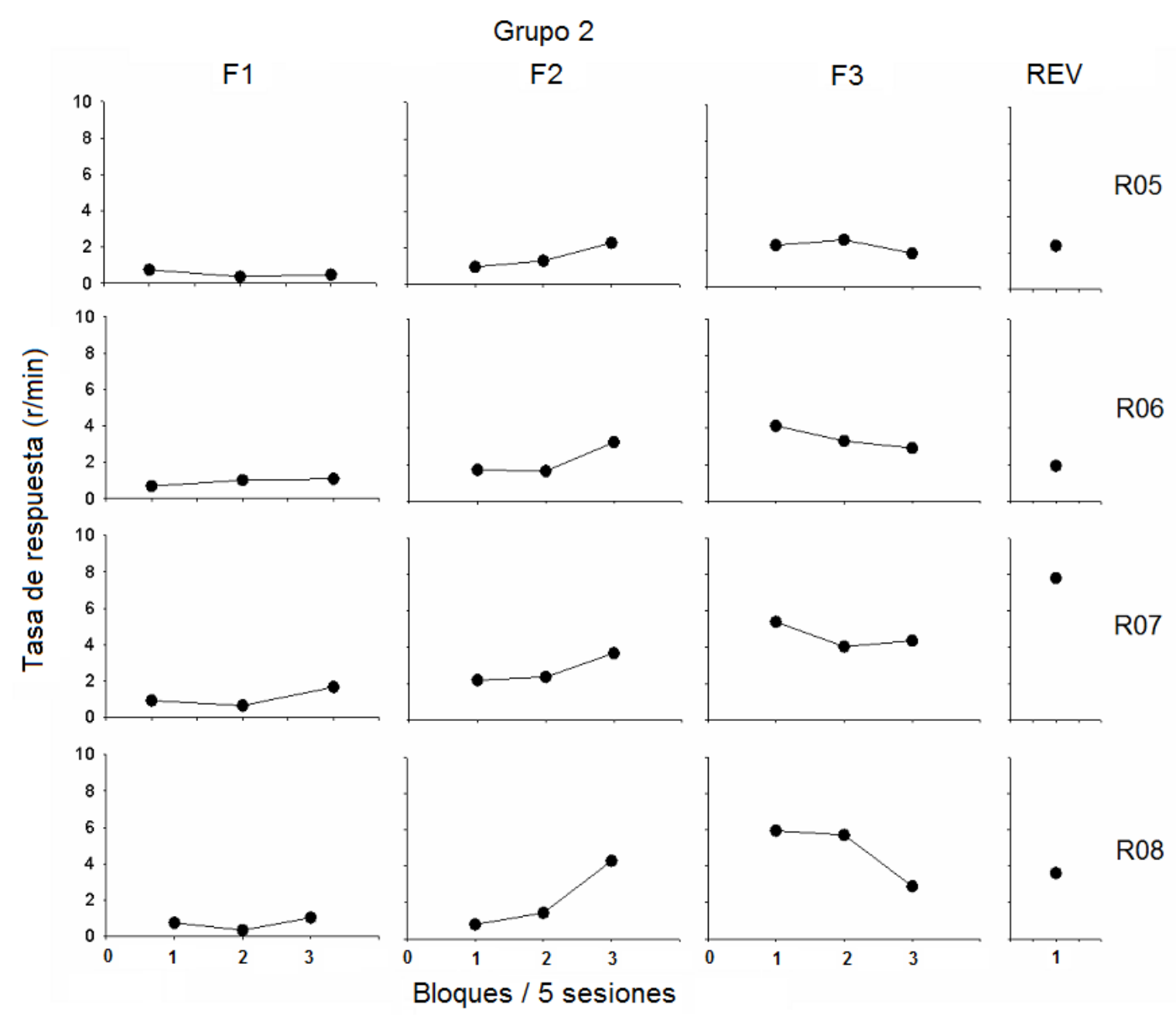

Figura 6. Tasa de respuesta promedio (respuestas por minuto) para las ratas del Grupo 2 a través de bloques de cinco sesiones. Para la fase de reversión solo se tomaron en consideración las primeras cinco sesiones. $\mathrm{R}$ es rata, $\mathrm{F}$ es fase y REV es reversión.

En la Figura 7 se muestra la tasa de respuesta promedio (respuestas por minuto) para el Grupo 1 (panel superior) y el Grupo 2 (panel inferior) a través de las tres primeras fases. Los datos de la fase de reversión fueron excluidos con el fin de conformar grupos comparables para el análisis estadístico. El Grupo 1 mostró tasas de respuestas similares durante las primeras dos fases, manteniéndose con una media cercana a 4 respuestas. En la Fase 3, este grupo mostró un incremento, en comparación con las fases previas, alcanzando las 6 respuestas en promedio. Se realizó un análisis con la prueba Friedman con el propósito de determinar si existían diferencias en las tasas de respuesta del Grupo 1 presentadas en la Fase $1(M d n=3,416)$, Fase $2(M d n=3,883)$ y Fase $3(M d n=5,133)$. La prueba reveló diferencias entre fases, $\chi^{2}(2, N=4)=40,412, p=0,001, W=0,337$, y el análisis post hoc por medio de la prueba DunnBonferroni identificó diferencias entre la Fase 1 y la Fase $3(0,001)$, y entre la Fase 2 y la Fase $3(0,001)$ después del ajuste de Bonferroni. No se encontraron otras diferencias entre las fases. En conjunto con esto, se empleó la prueba de Wilcoxon para confirmar la existencia de diferencias entre las condiciones con valores extremos en lo que respecta a la distancia espacial entre el conjunto luz-palanca y el bebedero, es decir, la Fase 1 y la Fase 3. Este análisis reveló que existen diferencias entre las tasas de respuesta, $Z(N=4)=-4,623$, $p=0,001, d=0,93$, presentadas por el Grupo 1 en la Fase $1(M d n=3,416)$ y la Fase $3(M d n=5,133)$. 


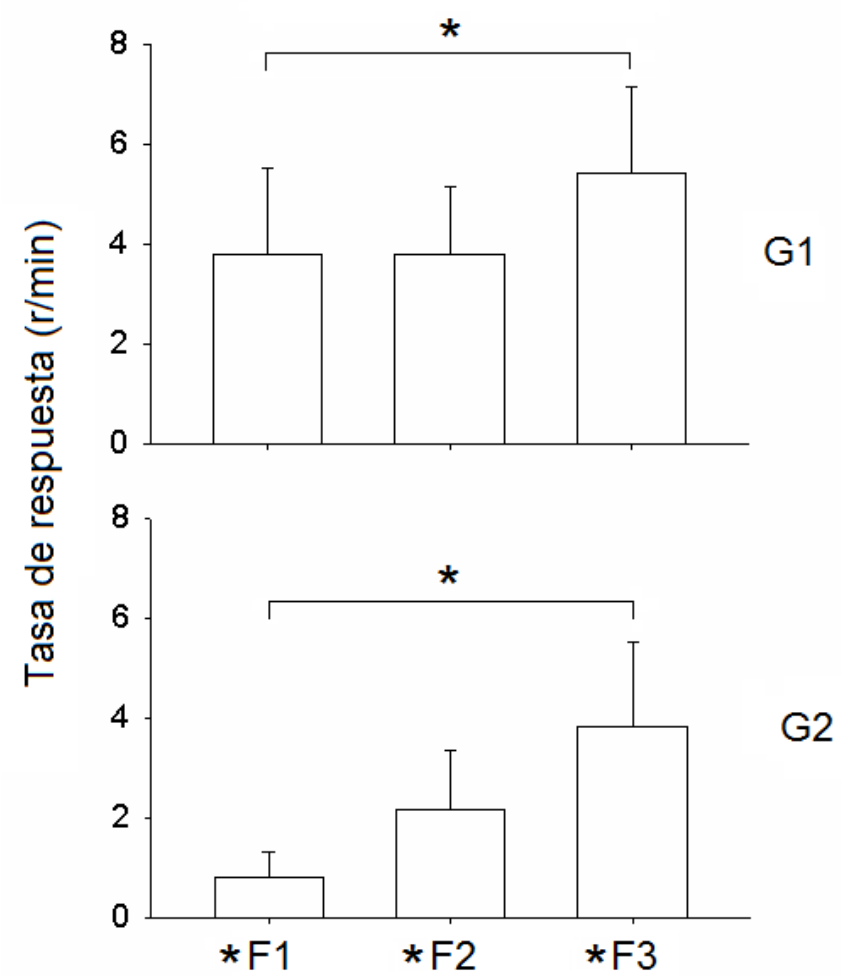

Figura 7. Tasa de respuesta promedio (respuestas por minuto) para el Grupo 1 (panel superior) y el Grupo 2 (panel inferior) a través de las tres primeras fases. $\mathrm{G}$ es grupo y $\mathrm{F}$ es fase. $\mathrm{El}$ asterisco indica que se encontraron diferencias por medio de la prueba de Wilcoxon. El asterisco adjunto a la $\mathrm{F}$ de fase indica que se encontraron diferencias con la prueba $U$ de Mann-Whitney. Las barras de error representan la desviación estándar.

El análisis para comparar las tasas de respuesta en el Grupo 2 identificó que existen diferencias entre la Fase $1(M d n=0,700)$, la Fase $2(M d n=2,050)$ y la Fase $3(M d n=3,616), \chi^{2}(2, N=4)=83,318, p=0,001$, $W=0,686$. Por su parte, el análisis post hoc por medio de la prueba Dunn-Bonferroni reveló diferencias entre la Fase 1 y la Fase $2(0,001)$, entre la Fase 2 y la Fase $3(0,008)$ y entre la Fase 1 y la Fase $3(0,001)$ después del ajuste de Bonferroni. La prueba de Wilcoxon para confirmar las diferencias entre las condiciones con valores extremos en la distancia espacial entre el bebedero y el conjunto luz-palanca reveló diferencias entre las tasas de respuesta, $Z(N=4)=-6,736, p=0,001, d=2,012$, presentadas por el Grupo 2 en la Fase $1(M d n=0,700)$ y la Fase $3(M d n=3,616)$.

Con el objetivo de comparar las tasas de respuesta presentadas por los grupos en cada fase se empleó la prueba $U$ de Mann-Whitney. Para la Fase 1, se encontró que existen diferencias, $Z(N=8)=-9,176, p=0,001$, $d=2,338$, entre el Grupo $1(M d n=3,416)$ y el Grupo $2(M d n=0,700)$. En la Fase 2 también se encontraron diferencias al comparar las tasas de respuesta del Grupo $1(M d n=3,883)$ y el Grupo $2(M d n=2,050), Z(N=8)$ $=-5,889, p=0,001, d=1,279$. Finalmente, durante la Fase 3, se presentaron diferencias en la tasas de respuesta, $Z(N=8)=-4,658, p=0,001, d=0,919$, entre el Grupo $1(M d n=5,133)$ y el Grupo $2(M d n=3,616)$.

\section{Discusión}

El propósito de este trabajo fue explorar los efectos de variar la distancia espacial entre el bebedero y el conjunto luz-palanca sobre la ubicación espacial y la tasa de respuesta en ratas. El desarrollo de este trabajo siguió la lógica de los programas definidos temporalmente (Schoenfeld \& Cole, 1972; Schoenfeld et al., 1956); no obstante, los parámetros temporales incluidos en este tipo de metodología se mantuvieron 
estables, debido a que los procedimientos propuestos en el presente trabajo se encuentran en una etapa incipiente. Además, el interés del experimento aquí descrito se concentró en los efectos de manipular alguna propiedad espacial de la ocurrencia de los estímulos. Es así que cobra sentido mantener los parámetros temporales fijos con el fin de controlar sus posibles efectos sobre la actividad de los sujetos. Sin embargo, es posible que en el futuro el tipo de manipulaciones que se describen en este trabajo se empleen en combinación con los procedimientos que tradicionalmente caracterizan a los programas temporales.

Los resultados obtenidos parecen ser congruentes con los hallazgos encontrados en otras áreas de investigación. En relación con la ubicación espacial en las zonas contiguas a los dispositivos de estímulo y respuesta, las ratas del Grupo 1 permanecieron más tiempo en la zona contigua al bebedero. Esta permanencia cambió de una zona a otra, de acuerdo al lugar en el que se instaló el bebedero en cada fase. Los cambios en la ubicación espacial de las ratas presentaron el siguiente orden: Zona 1 -Zona 2 - Zona 3 - Zona 1. Por otra parte, las ratas del Grupo 2 mostraron el mismo efecto, pero en orden inverso, es decir, Zona 3 - Zona 2 - Zona 1 - Zona 3. Estos hallazgos cobran sentido si se considera que en la Fase 1 para el Grupo 1 el bebedero se ubicó en el puerto contiguo a la Zona 1, mientras que para el Grupo 2 se instaló en el puerto contiguo a la Zona 3. De forma grupal, en la Figura 4 se comparó la ubicación espacial mantenida en una sola zona (ZB+) con la ubicación espacial mantenida en dos zonas (ZB-), demostrando que las ratas permanecieron más tiempo en la zona (ZB+) contigua al bebedero.

Este efecto se relaciona con los resultados obtenidos por Baum y Rachlin (1969). En ese estudio, palomas fueron expuestas a condiciones experimentales en las que los reforzadores fueron entregados en dos ubicaciones espaciales diferentes. Los autores reportaron que la razón de tiempo que las palomas pasaron en uno de los dos lados de la cámara experimental fue directamente proporcional a la razón de reforzamiento producida en cada dispensador. Efectos similares a este han sido encontrados en otros estudios (Pear \& Rector, 1979; Pear, 1985; Ribes \& Torres, 2000; Ribes, Torres, Correa, \& Montes, 2006). Empero, en estos experimentos la distancia espacial entre los estímulos (palanca, luz, bebedero) no varió, en otras palabras, la dimensión espacial de los estímulos no fue manipulada en el sentido de variable independiente. A pesar de las diferencias entre los procedimientos empleados por Baum y Rachlin (1969) y los que se emplearon en este trabajo, es posible afirmar que los resultados de ambos revelan el mismo efecto. En concreto, la ubicación espacial del organismo depende más del componente motivacional de la respuesta de consumo (Timberlake \& Lucas, 1985) que del número de estímulos presentes en la cámara experimental o de si estos cambian de ubicación. Otros hallazgos también brindan sustento a esta afirmación, por ejemplo, en algunos estudios en los que se ha empleado el laberinto acuático de Morris para generar el fenómeno de ensombrecimiento. El procedimiento general empleado para este propósito consiste en promover la saliencia de un estímulo, presentándolo de forma contigua a la plataforma (meta) que permite al sujeto escapar del agua. En estos estudios se ha encontrado que el organismo permanece más tiempo en el cuadrante o área en la que se encuentra la plataforma (Chamizo et al., 2006; Chamizo \& Rodrigo, 2004).

Los resultados sobre la tasa de respuesta mostraron niveles bajos para todas las ratas a lo largo del experimento. Este efecto puede ser explicado por la demora de reforzamiento. Al estudiar esta variable, se ha encontrado que la tasa de respuesta es una función inversa de incrementar el intervalo entre la respuesta procuradora y la entrega del reforzador (Lattal, 1987; Pulido Rull, Sosa Sánchez \& Valadez Téllez, 2006; Renner, 1964; Tarpy \& Sawabini, 1974). A pesar de que en este estudio no se manipuló la demora de reforzamiento, es posible suponer que, al mantener fijos los parámetros T, P y Ť, se pudo haber generado, de forma implícita, una demora. Por ejemplo, si la rata respondió al inicio del ciclo, la demora incrementa debido a que el agua se entregó al final del ciclo. En los estudios sobre demora de reforzamiento este tipo de procedimientos son conocidos como de demora variable (Lattal \& Gleeson, 1990). En adición, al identificar el control que el condicionamiento pavloviano ejerce sobre el comportamiento observado en las ratas de este estudio, puede plantearse que la demora que se crea cuando el organismo se traslada del conjunto luz-palanca al bebedero produce un condicionamiento de huella, caracterizado por ejecuciones más débiles, dependiendo del intervalo entre el estímulo condicional e incondicional (Pavlov, 1926/1927). Los efectos de la discontigüidad temporal entre estímulos en investigaciones que exploraron la dimensión espacial del condicionamiento pavloviano han sido discutidos tratando de identificar de forma aislada los efectos de la discontigüidad espacial (Christie, 1996). No obstante, para estudios en los que se emplee el procedimiento de la operante libre (Ferster \& Skinner, 1957) aislar los efectos de la discontigüidad espacial entre estímulos implica un reto, debido al desplazamiento del organismo a lo largo de la cámara 
experimental. Una posible alternativa a este problema consiste en realizar estudios con ensayos discretos en los que el organismo permanezca relativamente inmóvil ante un panel operativo (Murphy \& Miller, 1955).

$\mathrm{Al}$ comparar las tasas de respuesta grupales, se observó que, de forma general, las ratas presentaron un nivel bajo de respuestas, lo que podría relacionarse con el tamaño de la cámara experimental empleada. En estudios previos se ha encontrado que la actividad de los organismos ante los dispositivos de estimulación es una función inversa del tamaño o extensión de la cámara experimental (Serrano, 2013; Skuban \& Richardson, 1975). Considerando que las cámaras empleadas en este estudio son más grandes que las cajas de condicionamiento empleadas tradicionalmente en el AEC, haber encontrado tasas de respuesta bajas resulta congruente con hallazgos previos. A pesar de los niveles bajos en las tasas de respuesta, algunos efectos fueron encontrados. Por ejemplo, el Grupo 2 mostró valores bajos en comparación con el Grupo 1 durante la Fase 1, mientras que las tasas para el Grupo 2 fueron aumentando al pasar a las Fases 2 y 3, es decir, al ir reduciendo la distancia entre los estímulos, y las tasas para el Grupo 1 se mantuvieron relativamente estables o presentaron un ligero incremento al pasar a la Fase 3. El aumento progresivo en la tasa de respuesta para el Grupo 2 podría explicarse aceptando que la distancia espacial entre los estímulos implica una demora mayor en la entrega del reforzador, debido a que la rata debe recorrer de un lugar a otro (Christie, 1996). Como se ha mencionado antes, a mayor demora de reforzamiento menor tasa de respuesta.

Otra explicación para este efecto se relaciona con el estudio realizado por Baum (1982), en el que encontró que cuando dos programas de reforzamiento están disponibles al mismo tiempo, la distancia de desplazamiento de una palanca a otra impone un costo para el cambio de lugar. Sobre esta lógica, a mayor distancia de desplazamiento, menos recorridos de un lugar a otro y, por lo tanto, menores oportunidades de responder presionando la palanca. Esta formulación es congruente con los resultados obtenidos en estudios sobre aprendizaje discriminativo: la ejecución de los organismos es una función de incrementar la contigüidad espacial entre los estímulos (Iwai et al., 1986; Murphy \& Miller, 1955, 1959; Polidora \& Fletcher, 1964; Stollnitz, 1965; Yaginuma \& Iwai, 1986). Sin embargo, estas explicaciones no pueden ser aceptadas por completo, debido a que las ratas del Grupo 1 no mostraron un decremento en las tasas de respuesta al aumentar la distancia entre los estímulos. Esto sugiere que el orden en el que las ratas fueron expuestas a las condiciones experimentales (aumentar o disminuir la distancia espacial entre el bebedero y el conjunto luz-palanca) afectó la tasa de respuesta, independientemente de que la distancia espacial haya sido la misma para ambos grupos (Fase 2).

De forma general, los estudios en los que se emplean programas definidos temporalmente siguen una lógica paramétrica en la que, habiendo identificado de forma confiable los efectos de una variable, se van manipulando sistemáticamente diversos valores de esta variable para conocer sus efectos sobre el comportamiento de los organismos. De este modo, se han identificado parámetros como $\mathrm{t}^{\mathrm{D}}$ o $\mathrm{t}^{\Delta}$ y las preparaciones experimentales se han caracterizado por la manipulación de variables que determinan la ocurrencia de los estímulos en coordinadas temporales como, por ejemplo, la duración del ciclo $\mathrm{T}$ o la probabilidad de reforzamiento. En el presente trabajo se propuso la integración del estudio de la dimensión espacial, como determinante de los fenómenos conductuales, a esta lógica paramétrica. Siguiendo este objetivo, a continuación se presenta una lista con algunas propiedades espaciales que podrían ser estudiadas en investigaciones futuras que empleen procedimientos similares a los descritos en este trabajo. A pesar de que esta lista se basa en los parámetros incluidos en los programas definidos temporalmente, no representa un sistema nuevo; más bien es una descripción de variables que pueden ser agregadas al sistema ya conocido:

1. (U) La ubicación cardinal de un estímulo relativa a otra coordenada espacial. Esta variable hace referencia a la ubicación (norte, sur, oriente o poniente) de un estímulo en relación a otro punto, por ejemplo, en estudios tradicionales con cajas de condicionamiento operante un estímulo visual podría ubicarse al norte del manipulandum.

2. (D) Distancia de la ocurrencia de un estímulo relativa a otra coordenada. Siguiendo con el ejemplo dado para el caso anterior, esta variable puede ser identificada como los centímetros o pulgadas entre el estímulo visual y el manipulandum.

3. (O) El orden de ocurrencia de un estímulo en relación a la ocurrencia de un estímulo anterior. Esta variable ha sido estudiada usando la tarea de aprendizaje tiempo-lugar. Como se describió en el primer apartado de este artículo, en dichos estudios el alimento se entrega en dispositivos ubicados en diferentes lugares en orden secuencial o aleatorio. 
4. (Dg) El tamaño o la distribución geográfica que ocupa un estímulo. La ejecución de un organismo puede verse afectada por el tamaño de un estímulo, por ejemplo, en estudios sobre aprendizaje espacial empleando el laberinto acuático de Morris, una señal grande puede generar mayor saliencia que una pequeña.

5. (Dc) El tamaño o distribución geográfica del campo. Esta variable se relaciona a la extensión geográfica en la que el sujeto se puede desplazar, es decir, el tamaño de la cámara experimental.

\section{Referencias}

Arnau, J. (1990). Diseños experimentales en psicología y educación (2a ed., Vol 2). México DF, México: Trillas.

Baum, W. M. (1982). Choice, changeover, and travel. Journal of the Experimental Analysis of Behavior, 38, 35-49. https://doi.org/10.1901/jeab.1982.38-35

Baum, W. M. \& Rachlin, H. C. (1969). Choice as time allocation. Journal of Experimental Analysis of Behavior, 12 , 861-874. https://doi.org/10.1901/jeab.1969.12-861

Biebach, H., Gordijn, M. \& Krebs, J. R. (1989). Time-and-place learning by garden warblers, Sylvia borin. Animal Behaviour, 37, 353360. https://doi.org/10.1016/0003-3472(89)90083-3

Blaisdell, A. P., Schroeder, J. E. \& Fast, C. D. (2018). Spatial integration during performance in pigeons. Behavioural Processes, 154, 73-80. https://doi.org/10.1016/j.beproc.2017.12.012

Carr, J. A. R. \& Wilkie, D. M. (1998). Characterization of the strategy used by rats in an interval time-place learning task. Journal of Experimental Psychology: Animal Behavior Processes, 24, 151-162. https://doi.org/10.1037/0097-7403.24.2.151

Castro, L. (1977). Diseño experimental sin estadística: usos y restricciones en su aplicación a las ciencias de la conducta (2ª ed.). México DF, México: Trillas.

Chamizo, V. D., Manteiga, R. D., Rodrigo, T. \& Mackintosh, N. J. (2006). Competition between landmarks in spatial learning: The role of proximity to the goal. Behavioural Processes, 71, 59-65. https://doi.org/10.1016/j.beproc.2005.11.003

Chamizo, V. D. \& Rodrigo, T. (2004). Effect of absolute spatial proximity between a landmark and a goal. Learning and Motivation, 35, 102-114. https://doi.org/10.1016/S0023-9690(03)00059-6

Christie, J. (1996). Spatial contiguity facilitates Pavlovian conditioning. Psychonomic Bulletin \& Review, 3, 357-359. https://doi.org/10.3758/BF03210760

Ferster, C. B. \& Skinner, B. F. (1957). Schedules of reinforcement. New York, NY: Appleton-Century-Crofts. https://doi.org/10.1037/10627-000

Gould, J. L. (1987). Honey bees store learned flower-landing behaviour according to time of day. Animal Behaviour, 35, $1579-1581$. https://doi.org/10.1016/S0003-3472(87)80038-6

Harrison, J. M. \& Breed, M. D. (1987). Temporal learning in the giant tropical ant, Paraponera clavata. Physiological Entomology, 12, 317-320. https://doi.org/10.1111/j.1365-3032.1987.tb00756.x

Iversen, I. H., Sidman, M. \& Carrigan, P. (1986). Stimulus definition in conditional discriminations. Journal of the Experimental Analysis of Behavior, 45, 297-304. https://doi.org/10.1901/jeab.1986.45-297

Iwai, E., Yaginuma, S. \& Mishkin, M. (1986). Acquisition of discrimination learning of patterns identical in configuration in macaques (Macaca mulatta and M. fuscata). Journal of Comparative Psychology, 100, 30-36. https://doi.org/10.1037/0735-7036.100.1.30

Lattal, K. A. (1987). Considerations in the experimental analysis of reinforcement delay. En M. L. Commons, J. E. Mazur, J. A. Nevin $\&$ H. Rachlin (Eds.), Quantitative analysis of behavior. Vol. V: The effect of delay and of intervening events on reinforcement value (pp. 107-123). Hillsdale, NJ: Lawrence Erlbaum.

Lattal, K. A. \& Gleeson, S. (1990). Response acquisition with delayed reinforcement. Journal of Experimental Psychology: Animal Behavior Processes, 16, 27-39. https://doi.org/10.1037/0097-7403.16.1.27

Leising, K. J., Ruprecht, C. M. \& Stahlman, W. D. (2014). Modulation of variation by response-reward spatial proximity. International $\begin{array}{lllll}\text { Journal of } & \text { Comparative } & \text { 27, } & 326-337 . & \text { Extraído }\end{array}$ https://scholar.umw.edu/cgi/viewcontent.cgi?article=1012\&context=psychological_science

Maes, J. H. R., Fontanari, L. \& Regolin, L. (2009). Spatial reorientation in rats (Rattus norvegicus): Use of geometric and featural information as a function of arena size and feature location. Behavioural Brain Research, 201, $285-291$. https://doi.org/10.1016/j.bbr.2009.02.026

McClearn, G. E. \& Harlow, H. F. (1954). The effect of spatial contiguity on discrimination learning by rhesus monkey. Journal of Comparative and Physiological Psychology, 47, 391-394. https://doi.org/10.1037/h0059728

Morris, R. (1984). Developments of a water-maze procedure for studying spatial learning in the rat. Journal of Neuroscience Methods, 11, 47-60. https://doi.org/10.1016/0165-0270(84)90007-4

Mulder, C. K., Gerkema, M. P. \& Van der Zee, E. A. (2013). Circadian clocks and memory: Time-place learning. Frontiers in Molecular Neuroscience, 6, article 8. https://doi.org/10.3389/fnmol.2013.00008

Murphy, J. V. \& Miller, R. E. (1955). The effect of spatial contiguity of cue and reward in the object-quality learning of rhesus monkeys. Journal of Comparative and Physiological Psychology, 48, 221-224. https://doi.org/10.1037/h0045441

Murphy, J. V. \& Miller, R. E. (1959). Spatial contiguity of cue, reward, and response in discrimination learning by children. Journal of Experimental Psychology: General, 58, 485-489. https://doi.org/10.1037/h0041157

Nasser, H. M. \& Delamater, A. R. (2016). The determining conditions for Pavlovian learning: Psychological and neurobiological considerations. En R. A. Murphy \& R. C. Honey (Eds.), The Wiley handbook on the cognitive neuroscience of learning (pp. 7-46). Malden, MA: John Wiley \& Sons.

Pagano, R. R. (2011). Estadística para las ciencias del comportamiento (9a ed.). México DF, México: Cengage Learning.

Pavlov, I. P. (1926/1927). Conditioned reflexes: an investigation of the physiological activity of the cerebral cortex (G. V. Anrep, Trad.). London, Reino Unido: Oxford University Press.

Pear, J. J. (1985). Spatiotemporal patterns of behavior produced by variable-interval schedules of reinforcement. Journal of the Experimental Analysis of Behavior, 44, 217-231. https://doi.org/10.1901/jeab.1985.44-217 
Pear, J. J. \& Rector, B. L. (1979). Constituents of response rate. Journal of the Experimental Analysis of Behavior, $32,341-362$. https://doi.org/10.1901/jeab.1979.32-341

Polidora, V. J. \& Fletcher, H. J. (1964). An analysis of the importance of S-R spatial contiguity for proficient primate discrimination performance. Journal of Comparative and Physiological Psychology, 57, 224-230. https://doi.org/10.1037/h0043928

Pulido Rull, M. A., Sosa Sánchez, R. \& Valadez Téllez, L. (2006). Adquisición de la operante libre bajo condiciones de reforzamiento demorado: una revisión. Acta Comportamentalia, 14, 5-21. Extraído de https://www.redalyc.org/pdf/2745/274520148001.pdf

Reebs, S. G. (1996). Time-place learning in golden shiners (Pisces: Cyprinidae). Behavioural Processes, 36, 253-262. https://doi.org/10.1016/0376-6357(96)88023-5

Renner, K. E. (1964). Delay of reinforcement: A historical review. Psychological Bulletin, 61, 341-361. https://doi.org/10.1037/h0048335

Rescorla, R. A. \& Cunningham, C. L. (1979). Spatial contiguity facilitates Pavlovian second-order conditioning. Journal of Experimental Psychology: Animal Behavior Processes, 5, 152-161. https://doi.org/10.1037/0097-7403.5.2.152

Ribes Iñesta, E. (1992). Sobre el tiempo y el espacio psicológicos. Acta Comportamentalia, 0(1), 71-84. Extraído de http://www.revistas.unam.mx/index.php/acom/article/download/18253/17350

Ribes-Iñesta, E., Palacios, H., Hernández, V. \& León, A. (2018). Effects of concurrent temporal and spatial allocation of water delivery on water-seeking behavior in rats. International Journal of Comparative Psychology, 31, artículo 6. Extraído de https://escholarship.org/uc/item/5dw0c197

Ribes, E., \& Torres, C. (2000). The spatial distribution of behavior under varying frequencies of temporally scheduled water delivery. Journal of the Experimental Analysis of Behavior, 73(2), 195-209. https://doi.org/10.1901/jeab.2000.73-195

Ribes, E., Torres, C., Correa, L., \& Montes, E. (2006). Effects of concurrent random-time schedules on the spatial distribution of behavior in rats. Behavioural Processes, 73, 41-48. https://doi.org/10.1016/j.beproc.2006.02.001

Rodrigo, T., Gimeno, E., Ayguasanosa, M. \& Chamizo, V. D. (2014). Navigation with two landmarks in rats (Rattus norvegicus): The role of landmark salience. Journal of Comparative Psychology, 128(4), 378-386. https://doi.org/10.1037/a0036544

Saksida, L. M. \& Wilkie, D. M. (1994). Time-of-day discrimination by pigeons, Columba livia. Animal Learning \& Behavior, 22, 143154. https://doi.org/10.3758/BF03199914

Schoenfeld, W. N. \& Cole, B. K. (1972). Stimulus schedules: The t-T systems. New York, NY: Harper \& Row.

Schoenfeld, W. N., Cumming, W. W. \& Hearst, E. (1956). On the classification of reinforcement schedules. Proceedings of the National Academy of Sciences, 42, 563-570. https://doi.org/10.1073/pnas.42.8.563

Schoenfeld, W. N. \& Farmer, J. (1970). Reinforcement schedules and the "behavior stream". En W. N. Schoenfeld (Ed.), The theory of reinforcement schedules (pp. 215-245). New York, NY: Appleton-Century-Crofts.

Serrano, M. (2013). Efectos de tres tipos de entrega de agua sobre el ajuste comportamental. Acta Comportamentalia, 21, $273-283$. Extraído de http://pepsic.bvsalud.org/pdf/actac/v21n3/a01.pdf

Sidman, M. (1960/1973). Tácticas de investigación científica. Evaluación de datos en experimentales en psicología (M. Sidman, Trad.; Título original: Tactics of Scientific Research: Evaluating Experimental Data in Psychology). Barcelona, España: Fontanella.

Sidman, M. (2000). Equivalence relations and the reinforcement contingency. Journal of the Experimental Analysis of Behavior, 74, 127-146. https://doi.org/10.1901/jeab.2000.74-127

Skinner, B. F. (1938). The behavior of organisms. New York, NY: Appleton-Century-Crofts.

Skinner, B. F. (1956). A case history in scientific method. American Psychologist, 11, 221-233. https://doi.org/10.1037/h0047662

Skuban, W. E. \& Richardson, W. K. (1975). The effect of the size of the test environment on behavior under two temporally defined schedules. Journal of the Experimental Analysis of Behavior, 23, 271-275. https://doi.org/10.1901/jeab.1975.23-271

Stollnitz, F. (1965). Spatial variables, observing responses, and discrimination learning sets. Psychological Review, 72, $247-261$. https://doi.org/10.1037/h0022005

Swisher, M. \& Urcuioli, P. J. (2013). Symmetry in the pigeon with sample and comparison stimuli in different locations. Journal of the Experimental Analysis of Behavior, 100(1), 49-60. https://doi.org/10.1002/jeab.31

Swisher, M. \& Urcuioli, P. J. (2015). Symmetry in the pigeon with sample and comparison stimuli in different locations. II. Journal of the Experimental Analysis of Behavior, 104, 119-132. https://doi.org/10.1002/jeab.162

Tarpy, R. M. \& Sawabini, F. L. (1974). Reinforcement delay: A selective review of the last decade. Psychological Bulletin, 81, 984-997. https://doi.org/10.1037/h0037428

Timberlake, W. \& Lucas, G. A. (1985). The basis of superstitious behavior: Change contingency, stimulus substitution, or appetitive behavior? Journal of Experimental Analysis of Behavior, 44, 279-299. https://doi.org/10.1901/jeab.1985.44-279

Wong, J., Leising, K. J. \& Blaisdell, A. P. (2016). Spatial overshadowing in pigeons: Evidence for an acquisition deficit. International Journal of Comparative Psychology, 29, research article 1. Extraído de https://escholarship.org/uc/item/2p1212n7

Yaginuma, S. \& Iwai, E. (1986). Effect of small cue-response separation on pattern discrimination in macaques (Macaca fuscata and M. mulatta). Journal of Comparative Psychology, 100, 137-142. https://doi.org/10.1037/0735-7036.100.2.137

Fecha de recepción: Febrero de 2019.

Fecha de aceptación: Diciembre de 2019. 